\title{
Professional competencies formation in the field of aesthetic artwork evaluation
}

\author{
Irina Vlasova ${ }^{1, *}$, Yulia Ushanyova $^{2}$, Sergey Pisarenko ${ }^{2}$ \\ ${ }^{1}$ Don State Technical University, Rostov-on-Don, Russia \\ ${ }^{2}$ Southern Federal University, Rostov-on-Don, Russia
}

\begin{abstract}
The purpose of the article is a brief description of the author's method for solving the problem of professional competencies formation for students studying in art and adjoining specialties related to the ability to carry out aesthetic evaluation of artworks and independently conduct educating and educational activities. This problem is particularly relevant in modern conditions, which are characterized by high transmission speeds of a large amount of visual information. The methodology is based on a system of forming professional tools for students to conduct analytical work in the field of aesthetic evaluation of artworks. The development of this methodology was carried out in accordance with modern didactic principles, such as the principles of science, the relationship between theory and practice, as well as the principle of visibility. These principles were transmitted at various levels of the educational process organization. To collect information, we used such methods as analyzing literary sources on the research problem, conducting surveys of students and teachers, and analyzing modern resources for storing and distributing images, namely, author's works of art. The educational technologies described in the article can be applied in the process of training specialists in the field of architecture, all types of visual design, painting, graphics, sculpture, as well as specialists in the field of art and correctional pedagogy, art therapy.
\end{abstract}

\section{Introduction}

Modern requirements for the training of art specialists include a number of professional competencies that are responsible for their general cultural and aesthetic training. This attitude is reflected in the educational standards of the Russian Federation and many other countries. The ability to analyze an art work from the point of view of aesthetic context is directly related to two aspects of the work of a professional artist:

- practical work in a particular form of visual art, including knowledge of the material and modern visual forms, technologies;

- ability to analyze the work from the point of view of cultural and iconic context.

Speaking about the second aspect, it should be noted that in the context of this research, we turn to the iconographic approach, the main methods of which were formulated by representatives of the so-called Warburg school. This approach implies three levels of understanding of an art work: preiconographic analysis, iconographic analysis, and

\footnotetext{
* Corresponding author: pisarenko-21@yandex.ru
} 
iconological interpretation, as well as three stages of painting: phenomenal, meaningful and documentary.

Considering the problem of the image aesthetic context and its role in the training of artists, we turned to the works of J. Elkins [1]. Considering the psychophysiological features of image perception associated with the existing experience of artistic activity, we turned to the works of a number of authors, such as R. Gregory [2], S. Frith [3], D. Hoffman [4], E. Kandel [5, 6], E. Gombrich [7], M. Dow [8], N. Livingston [9], P. Mamassian [10]. In addition, a number of philosophical aspects of the visual arts development are considered in the publications of I. Verstegen [11] and M. Shapiro [12].

After analyzing these scientists' work, we can draw the following conclusions.

1. Modern human and art sciences, such as psychology, physiology, medicine, art history and art pedagogy, have a fairly wide range of methods and evidence-based material that confirms the relationship between the psychology of image perception and the aesthetic evaluation of an art work.

2. the research of the mechanisms of forming the skills of artwork aesthetic analysis lies in an interdisciplinary space that affects such aspects of visual activity as artistic practice, professional art education, the art market, the visual technologies development and the history of world art culture.

3. in modern research devoted to the art specialists training, the question of forming the skills of artwork aesthetic evaluation is raised only in the historical or art history context, when studying relevant disciplines, such as, for example, art history. However, modern artistic creativity is largely not limited to the academic concept of fine art, which indicates that there is a significant unresolved problem in the art pedagogy field that goes beyond the framework of a particular educational system.

4. this poses new challenges for art professionals related to rapid reflection and the ability to identify a high-quality product of artistic and creative activity.

Taking into account the above conclusions from the thematic analysis of the main sources on the subject of research, it is possible to define its problem as the lack of teaching methods in modern art professional education that allow students to independently tooling search for objective professional, aesthetic interpretation of fine art works.

Thus, the research hypothesis is the suggestion that the solution to the problem of the teaching methods lack in modern art professional education that allow students to independently tooling search for an objective professional, aesthetic interpretation of fine art works can be implemented by organizing the educational process in such a way that the synthesis of practical experience in conducting visual activities with a theoretical base would be optimal.

The research object is to consider the training process of art specialists to improve its effectiveness by developing new forms of synthesis between the theory and practice of educational activities. One of the conditions for implementing this model of the educational process, in our opinion, can be a formal approach to the visual characteristics of the image and an analysis of its organization in terms of aesthetic impact on the viewer.

In the context of this research, it is necessary to clarify the concept of visual creativity. This concept implies the differentiation of such terms as art, creativity, visual activity, artistic and creative activity. Thus, creative activity does not always correspond to the concept of "art", which implies a deeper approach to understanding this type of work and is more often associated with the presence of a high degree of performing skill or a pronounced conceptual and value content in the concept of a particular artistic culture. Artistic and creative activity is understood as the process of creating a fine art work. Thus, it is possible to build a semantic chain of concepts in which visual creativity (activity) can correspond to artistic and creative activity, that is, it can be synonymous with the process of creating an art work, or it can mean 
the creation of a product that carries visual information, but does not have aesthetic and cultural value.

The distinction between these concepts is especially relevant in modern conditions, which are characterized by rapidly expanding databases that contain a huge amount of visual information, including copyrighted works, the quality of which, from the point of view of a professional approach, is becoming increasingly difficult to judge unambiguously.

This kind of differentiation is the fundamental basis of professional art education, and allows students to set the task of finding mechanisms for displaying artworks in the modern information and visual space. Due to this problem understanding, our research actively uses such modern educational technology as the problem-based learning method, when a student is faced with the need to justify the work artistic value in comparison with other analogues. In other words, modern art education is faced not with the problem of the availability of new sources of visual information, but with their quality. As J. Elkins notes: "Visualization is not only a product of culture, but serves as its agent" [1, p. 5]. It is highly qualified specialists who will have the task of updating the most valuable experience of artistic activity and maintaining a high-quality level of artworks performance. In such difficult conditions, the educational process is currently taking place all over the world, so the problem of the claimed research is relevant and must be carefully studied and resolved.

In this context, the question arises about the scientific justification of the aesthetic evaluation of visual activity product. This question can be formulated somewhat differently: what professional knowledge and skills should a specialist have in order to make objective conclusions about the aesthetic value of a particular work? The solution of this issue is described using the author's methodology in the main part of the article. The formation of objective, scientifically-based tools in this case is the main professional skill of an art specialist. We consider an example of the application of a technique that teaches students of art universities to independently form tools for professional aesthetic assessment in the framework of the experiment described below.

\section{Materials and methods}

To describe the results of testing the methodological system for developing the ability of art students to independently develop tools for aesthetic evaluation of the visual activity product, consider an experiment conducted at the Academy of Architecture and Arts of the Southern Federal University and the school of architecture, design and arts of the Don State Technical University. The experiment involved first-and second-year students studying in the fields of "Graphic design", "Painting", "Graphics" and "Sculpture". The choice of groups was determined by the priority of the main tasks at this professional training stage, namely: improving the level of literacy in the field of visual theory, forming and consolidating basic visual skills, mastering the method of creating images, and getting acquainted with the expressive capabilities of various materials. Another reason for this choice is due to the fact that at this stage of training, according to surveys, students master about $90 \%$ of basic professional concepts.

Before conducting the experiment, a system of criteria was developed, which can be used to judge the formed tools that allow the student to give an objective, scientifically based, professional and aesthetic assessment of a fine art work. It should be noted here that due to the fact that most of the time spent is devoted to the practice of visual activity, it is advisable to form the skills of aesthetic image analysis in these classes, and not only in such theoretical lecture courses as, for example, art history.

For correct scientific criteria justification for the degree of formation of professional aesthetic image analysis skills, it is necessary to minimize the scope of the experiment and show the work of the author's methodology on the example of teaching a specific discipline, 
the choice of which should be justified by its universality for all areas of training. This discipline is traditionally considered academic drawing. For the experiment accuracy, we will limit ourselves to studying short-term forms of drawing made on the basis of full-scale material from memory, from nature, or from representation, namely, various types of sketches. These types of visual activities also develop associative capabilities for identifying objects. A well-known visual perception theorist R. Gregory notes that the image of a particular object is fixed in the mind at the level of sensations [2, p. 6]. A similar idea is put forward by a specialist in cognitive psychology K. Frit, who says that the world perception is largely a flight of fantasy, which nevertheless coincides with objectivity, and the human brain manages to combine signals with individual experience [3, p. 111].

So, in order to analyze the aesthetic value of a work made in the form of a sketch, it is necessary to develop a system of evaluation criteria that is relevant for this type of visual activity. To do this, we need to refer to the achievements of the iconographic method of image analysis, according to which two approaches can be defined: practical and theoretical.

The practical approach characterizes the performance features of a particular work:

- goals and objectives of this type artistic activity, i.e. what practical or creative tasks can be solved by this type of image. What opportunities it gives the artist, in terms of expressive fixation of reality.

- what means of visual expression are typical for this type of short-term academic drawing.

The theoretical approach characterizes an image in terms of meaning, image, or sign, as well as image stylistics:

- what period of world art culture development does the style of performance of a particular image belong to?

- what visual features characterize the uniqueness of the visual language of this work, what visual associations are used in this case.

Explaining the first approach to image analysis, which is practical, we should refer to the history of the academic drawing forms development. This is how long-term and short-term types of academic drawing have historically developed in the Russian academic tradition. The latter in European artistic practice are called sketch (English "sketch" - sketch, quick outline). In Russian art practice, this concept sounds more specific. So, a short - term drawing refers to 2 categories- sketches, outlines. At the same time, each of them has its own specific classification. For example, sketches are divided by the type of graphic means of expression, which include such formal categories as line, spot, and dot. Accordingly, there are different versions of linear and tonal sketches. There are also combined types of outlines that unite various means of graphic expression.

Explaining the second approach, which is theoretical, students should pay attention to the need to correlate the analyzed image according to the goals and objectives of performance, and accordingly the style, in the world art culture context. At the same time, it is not necessary to establish the authorship and exact time of this work creation, it is enough to determine the main styles of fine art and, accordingly, the place and time with which a particular image can be correlated. Also, on this stage is to undertake a comparative analysis of the images considered from the point of view of technique and means of expression with known similar works of famous masters of the era, that requires recourse to the knowledge that students gained in the classroom for art history. Also, the image theoretical analysis includes reading the associative context of the image. It can pass directly when the image is easy to read, and does not carry a sign context, does not require guessing. For example, the image is made in a realistic manner typical of Western European art since the 15th century. Or it requires additional effort to read, if it appeals more to the associative thinking of a person through the form stylization, which is more typical for traditional folk, stylized and decorative forms of the image. 
Thus, it is possible to determine the main sources of image analysis from the point of view of science and practice of conducting visual activities, which can be criteria for evaluating the aesthetic significance of a work, taking into account the categories of an interdisciplinary approach. It is with this approach that the aesthetic value of the image can be justified not at the household level, but at the professional level. The ability to develop a similar system of criteria applicable to a particular type of visual activity will indicate the students' ability to independently conduct a professional aesthetic assessment of the image.

Experimental work of the passage in two stages. The tasks of the first stage were as follows:

- identification of control and experimental groups;

- determining the degree of students initial training in the field of professional and aesthetic analysis of art work (in the specific case of sketches);

- conducting a survey of students to identify their understanding of the goals and objectives of sketches, knowledge of their classification, as well as individual stylistic preferences;

- conversations with leading teachers in the discipline "drawing" for optimal time synchronization of the studied material with the experiment goals and objectives;

- development and technical system preparation for maintaining statistical indicators based on the research results.

The tasks of the second stage consisted in carrying out the practical part of the experiment, recording and analyzing the data obtained as a result. Namely, when performing specific practical tasks presented in the framework of the methodological system, it becomes possible to obtain data to infer the degree of skills formation of professional visual work aesthetic analysis by students studying in the specialties "graphic design", "painting", "graphics", "sculpture".

Based on the results of the first part of the experiment, we identified 2 groups, each of which had 18 people. The first group - control, the second - experimental were formed from first-and second-year students who receive training in graphic design, easel and monumental painting, graphics, sculpture. At the same time, first-and second-year students were evenly divided into groups for the same average indicator for the levels of practical and theoretical training.

As a survey result, it was possible to identify approximately the same level of theoretical and practical groups' training in the field of sketches and outlines according to the above assessment criteria. Namely, the number of correct and complete answers in a specially prepared questionnaire in both groups turned out to be approximately the same, namely, about $15 \%$ of the possible ones, which refers to the first and lowest degree of formation of professional aesthetic image analysis skills. Also, as a result of the conversation with the students, it was possible to identify their preferences in the sketching style field and execution techniques. Thus, it became clear that students are most familiar with the forms of short term drawing typical of European art of the mid-19th and early 20th centuries, and are not well informed in the decorative folk-art field and traditional forms of short-term images for Asian and American countries. This allowed us to determine the thematic component of the practical lesson.

Conversations with leading teachers on the subject of "drawing" allowed us to establish that it is more appropriate to conduct practical exercises presented in the experiment for the first time directly in the classroom on academic drawing, before performing long tasks from nature.

The second part of the experiment was presented in two stages. At the first stage, students were offered to get acquainted with the types of sketches, then they were asked to perform certain types of sketches (linear sketch) in different graphic techniques from nature. In addition, students were asked to find and analyze fine art various examples, where a similar 
method of work was used. At this stage of working with examples, the teacher's role is great. It is his responsibility to select high-quality samples for research. At the same time, it is necessary to pay special attention to the fact that the samples are as diverse as possible. For example, to conduct a practical exercise, you need to make a selection of reproductions illustrating the art of Europe and Asia, especially Chinese traditional techniques (Xie-Yi Chinese painting), and the European tradition of rapid full-scale drawing performed in a few seconds (crocs). This is an excellent example to demonstrate to students the power of associative properties of thinking, its capabilities in the field of visual material synthesis, and its correlation with the existing experience of perception. This material also explains that along with the synthesis of visual associations in the viewer's mind, reverse processes occur, for example, focusing on some unique feature of nature that reflects the state of the depicted object. At this stage, it was important to explain to students that this ability to think is the basis for creating an expressive artistic image.

After completing the practical part of the task, students are asked to evaluate their work by compiling a table containing the main criteria by which they can evaluate their work quality. Also, during the conversation, they could express what they think they managed to convey in their own sketches, and what they did not, explain what goals and objectives they set when completing the drawing linear sketches task.

Then the students' sketches were analyzed in terms of identifying the practical level of performance. The following indicators were taken into account: the ability to convey the object proportions, use the tonal range of the line, compose the image, and identify the plastic movement of the form due to its design features.

The second stage of the experiment involved performing such work independently. At the same time, students were required to create the most diverse database of visual material from examples of the world's artistic heritage, where they could demonstrate graphic techniques variety for creating a quick image using various types of spots.

After that, students were also asked to perform types of sketches from nature using different types of spots, avoiding the use of lines in one form or another. After completing this work, students were given the task to independently develop a table where they could describe in detail the criteria for evaluating their work. Further, these sketches were also taken by the teacher to analyze their technical performance level.

In the control groups, sketches were taught in the traditional way, namely, after the teacher personally showed the main working techniques in graphic techniques, students began to perform sketches from nature. After completing the necessary series of sketches, the control group students were also asked to create a table in which they would give the evaluation criteria and the resulting sketches analysis.

A comparison of the number of points and their classification, given in the students table was a material on the basis of which to judge the effectiveness of the methodology employed in the experimental groups, as the number of items and their heterogeneity allow us to judge the degree of student readiness to conduct professional aesthetic image analysis, for example the short forms of the figure.

At this stage, 3 degrees of formation of professional aesthetic image analysis skills were determined (for example, sketches). Level 1 - up to $30 \%$ of the possible criteria, level 2 - up to $80 \%$ of the criteria, and level 3 - the highest over $80 \%$ of the criteria.

In our experimental work, we applied such didactic principles as the principle of science, which was manifested in the form of educational material presentation, in addressing various areas of scientific knowledge. Important in this method is the principle of visibility, which was used when the teacher personally showed drawing techniques, working from nature and demonstrating reproductions. But one of the most important didactic principles that implement the presented methodology is the principle of connection between theory and 
practice. Thus, students conducted practical and theoretical work simultaneously, directly being in an interdisciplinary educational model.

As a final task, which was supposed to be indicative of the effectiveness of the teaching methods used, students were instructed to create a table-description of any examples of shortterm drawing from the Pinterest database, randomly selected based on individual preferences. This digital resource provides an extensive database of images, photographs and reproductions.

\section{Results}

To systematize the research results, we developed the following calculation system. According to the developed criteria for evaluating the work, we noted the points that students of control and experimental groups considered important to indicate in their author's systems of aesthetic sketches' professional assessment. Based on the data provided in the tables, the level of training in this issue was almost the same at the first stage. Moreover, the control and experimental groups showed the same awareness of the goals and objectives of the outline due to the fact that second-year students, also evenly distributed in the control and experimental groups, were already prepared on this issue in the previous terms. Examples of aesthetic evaluation criteria developed by students in control and experimental groups are shown in Table 1.

Table 1. A list of characteristics specified by students when developing an independent system for evaluating the aesthetic qualities of images on the example of various types of sketches.

\begin{tabular}{|c|c|c|c|c|c|c|c|}
\hline \multirow{2}{*}{\multicolumn{2}{|c|}{$\begin{array}{c}\text { The main } \\
\text { positions of the } \\
\text { aesthetic image } \\
\text { analysis }\end{array}$}} & \multicolumn{2}{|c|}{1 task } & \multicolumn{2}{|c|}{2 task } & \multicolumn{2}{|c|}{3 task } \\
\hline & & \multirow{2}{*}{$\begin{array}{c}\begin{array}{c}\text { Contr } \\
\text { ol } \\
\text { group }\end{array} \\
+\end{array}$} & \multirow{2}{*}{$\begin{array}{c}\begin{array}{c}\text { Experiment } \\
\text { al group }\end{array} \\
+\end{array}$} & \multirow{2}{*}{$\begin{array}{r}\text { Contro } \\
\text { I group } \\
+\end{array}$} & \multirow{2}{*}{$\begin{array}{c}\begin{array}{c}\text { Experiment } \\
\text { al group }\end{array} \\
+\end{array}$} & \multirow{2}{*}{$\begin{array}{r}\text { Contro } \\
\text { l group } \\
+\end{array}$} & \multirow{2}{*}{$\begin{array}{c}\begin{array}{c}\text { Experiment } \\
\text { al group }\end{array} \\
+\end{array}$} \\
\hline \multirow{3}{*}{ 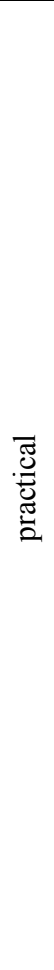 } & $\begin{array}{l}\text { Goals } \\
\text { (education } \\
\text { al and } \\
\text { creative) }\end{array}$ & & & & & & \\
\hline & $\begin{array}{c}\text { Tasks } \\
\text { (fixing the } \\
\text { proportion, } \\
\text { searching } \\
\text { for a } \\
\text { plastic } \\
\text { solution to } \\
\text { the form, } \\
\text { constructiv } \\
\text { e solution } \\
\text { of the } \\
\text { form, } \\
\text { studying } \\
\text { the } \\
\text { capabilitie } \\
\text { s of a } \\
\text { specific } \\
\text { technique) }\end{array}$ & + & + & + & + & + & + \\
\hline & $\begin{array}{l}\text { Technique } \\
\text { (definition } \\
\text { of image } \\
\text { execution } \\
\text { materials) }\end{array}$ & & & + & + & + & + \\
\hline
\end{tabular}




\begin{tabular}{|c|c|c|c|c|c|}
\hline & $\begin{array}{l}\text { Means of } \\
\text { image } \\
\text { expression } \\
\text { (in the } \\
\text { case of } \\
\text { sketches- } \\
\text { line, spot, } \\
\text { point) }\end{array}$ & + & + & + & + \\
\hline \multirow{4}{*}{ 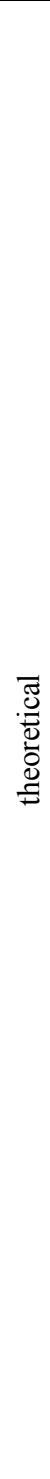 } & $\begin{array}{c}\text { What is } \\
\text { the image } \\
\text { stylistic } \\
\text { key }\end{array}$ & & + & + & + \\
\hline & $\begin{array}{l}\text { What time } \\
\text { frame is } \\
\text { the image } \\
\text { made in }\end{array}$ & & + & + & + \\
\hline & $\begin{array}{l}\text { What in } \\
\text { the image } \\
\text { contributes } \\
\text { to the } \\
\text { transfer of } \\
\text { the } \\
\text { depicted } \\
\text { object } \\
\text { emotional } \\
\text { character } \\
\text { (the nature } \\
\text { of the } \\
\text { pose, } \\
\text { means of } \\
\text { expression } \\
\text {, } \\
\text { proportion } \\
\text { s, shape of } \\
\text { the main } \\
\text { silhouette) }\end{array}$ & & & & + \\
\hline & $\begin{array}{l}\text { How high } \\
\text { is the } \\
\text { author's } \\
\text { performan } \\
\text { ce skill in } \\
\text { terms of } \\
\text { selecting } \\
\text { expression } \\
\text { means for } \\
\text { solving a } \\
\text { specific } \\
\text { artistic } \\
\text { image }\end{array}$ & & & & + \\
\hline
\end{tabular}

During the experiment, more than 300 sketches made in various graphic techniques were analyzed. So, after completing all three practical tasks, students were asked to make their own criteria list for aesthetic sketches assessment.

Thus, according to the first task results, students showed a low level of training in this issue, and only $25 \%$ of possible positions were indicated in the control and experimental groups. According to 3 degrees of professional aesthetic image analysis skills formation 
defined by us (for example, sketches), this corresponds to the lowest, level 1 - up to $30 \%$ of the possible criteria, level 2 - up to $80 \%$ of the criteria, and level 3 - over $80 \%$ of the criteria.

After completing the second task, there were quantitative changes in these assessments' indicators offered by students, and now they amounted to $50 \%$ in the control group, and $75 \%$ in the experimental group. After the third task, the number of grades offered by students in the experimental groups rose to $100 \%$, while a quarter higher than the control group, which was $75 \%$.

In general, in the experimental and control groups, a significant increase in indicators can be noted, and, characteristically, the last to be added to the evaluation criteria are theoretical ones related to the transfer of the characteristic features of the depicted object in the sketch and the artistic image creation. Thus, it can be concluded that students do not come to this understanding of image assessment immediately, but only on the basis of long-term work of both practical and theoretical nature.

These examples confirm the statistics obtained by us as a research result, according to which in the experimental group, students demonstrated knowledge of more criteria for evaluating a short-term drawing in terms of its aesthetic value as an independent work. At the same time, the number of proposed evaluation criteria in the experimental groups exceeded by almost a quarter, which indicates that students are more prepared in this field, which in turn confirms the proposed methodology effectiveness.

\section{Discussion}

Summing up the research results, it can be noted that the obvious increase in indicators in the experimental group is due to an integrated approach to training, which maximally involves interdisciplinary connections. The greatest difficulty in giving an aesthetic assessment of visual activity work (in this case, sketches) was caused by the image visual characteristics, which create an association necessary not only for identifying the object, but also for transmitting its internal characteristics, the state at the time of the image. The issue of transmitting emotions by means of visual art is difficult for beginners to learn the basics of visual literacy and for a wide range of the public. A vivid description of this practice is given in the work of K. Simson, dedicated to the art of Vienna, when the creative method of such expressive artists as Kokoschka and Schiele is understood by the public as an insult to the viewer [13].

This correlates with the primary goal of all art forms, namely, the artistic image creation. Nevertheless, thanks to the presented set of methods, students gradually develop professional thinking aimed at identifying image features that find the maximum aesthetic response from a wide range of the public. In his work "Artistic instinct", D. Dutton notes that art not only gives the most powerful emotional sensations, but also allows you to practice emotions and construct a certain empathic situation [14]. Thus, understanding the emotional characteristics of an artistic image is by no means reduced to the objective task of transmitting the maximum similarity even in works of a realist sense. The expression of Henri Matisse is interesting in this way, which is given in the work of X. Sparling dedicated to the work of this artist: "The appearance of photography made it possible for the artist to free himself from the need to copy nature and express emotions directly by the simplest means available" [15]. This circumstance also allows future specialists to be more competitive and develop new priority areas of the art market.

Aesthetic evaluation of artwork also assumes the most accurate, optimized way of expressing certain emotions from the artist's perception of the visual image, therefore, it can be considered as one of the aspects of work in the field of art therapy and correctional pedagogy. This technique introduction is also useful at the initial stages of fine arts education, 
in general education institutions, as well as in the additional education system, children's art schools for different age groups with different levels of initial training.

Despite the fact that this method was considered on the short-term drawing example, namely one of its forms, the basic principles of the experimental system set out in the article can be applied at various stages of professional training as different areas of training, such as architecture, painting, drawing, sculpture, design, when teaching various disciplines. For example, this method can increase the level of training of specialists in the painting and composition field.

It is worth noting that the explanation of some theoretical points in accordance with the proposed method takes quite a long time, and this reduces the amount of practical work on the drawing. However, such labor costs are relevant only at the initial stages of applying the methodology in the future, most of these tasks can be transferred to independent students work, which will increase their goals and objectives understanding of the educational process, and will also be an incentive for setting their own creative tasks. In addition, this approach helps to strengthen the connection of theoretical aspects of artistic and creative professional activity with practice.

\section{Conclusions}

The results of the experiment confirmed the proposed method effectiveness aimed at developing students studying in art specialties, the ability to aesthetic evaluation of fine art works (for example, working on sketches). The experimental groups students demonstrated a higher level both in the practical execution of various types of sketches, and in terms of the ability to carry out a theoretical analysis of the visual activity results. The following patterns were found:

- an important factor in the training process effectiveness using the experimental method was the variety of visual samples used, which makes it possible to reveal options for various visual approaches to working on sketches;

- the results of training according to the proposed method were positively affected by the training individualization carried out during the experimental work, which implies a comprehensive account of students' personal qualities;

- the interrelation and interdependence of students' theoretical and practical training in the field of short-term drawing was confirmed: familiarization with the fine art samples offered in the course of experimental work contributed to the expansion of students' theoretical ideas and the practical visual skills development; in turn, specially organized practical visual activities contributed to the development of the ability to carry out a reasoned aesthetic analysis of graphic works.

The results of the described experiment can be taken into account not only in relation to academic drawing, but also when conducting pedagogical research in related fields, in particular, in the field of teaching academic painting, composition and other disciplines.

\section{References}

1. J. Elkins, E. Feorintini, Visual Words. Looking. Images. Visual disciplines (Oxford university press, New York, 2020)

2. R. Gregory, Seeing Through illusions (Oxford University press, New York, 2009)

3. C. Frith, Making Up the Mind: How the Brain Greates Our Mental Word (Blackwell Publishing, Oxford, 2007)

4. D.D. Hoffman, Visual intelligence: How We Create What We See (W.W. Norton, New York, 1998) 
5. E.R. Kandel, Reductionism in Art and Brain Science: Bridging the Two Cultures (Columbia University Press, New York, 2016)

6. E.R. Kandel, The Age of Insight: the Quest to Understand the Unconscious in Art, Mind and Brain from Vienna 1900 to the Present (Random House, New York, 2012)

7. E.N. Gombrich, The Essential Gombrich: Selected Writings on Art and Culture (Phaidon Press, London, 1996)

8. N. Daw, How Vision Works (Oxford University Press, New York, 2012)

9. M. Livingstone, Vision and Art: The Biology of Seeing (Abrams, New York, 2002)

10. P. Mamassian, Vision Research 48, 2143 - 2153 (2008)

11. I. Verstegen, Journal of Art Historiography 15, 107 - 118 (2016)

12. M. Shapiro, Theory and Philosophy of Art: Style, Artist, and Society (George Braziller, New York, 1998)

13. K. Simpson, Journal of Art Historiography 3 (2010)

14. D.D. Dutton, The Art Instinct: Beaty, Pieasure, and Human Evolution (Bloomsbury Press, New York, 2009)

15. H. Spurling, Matisse the Master: A life of Henri Matisse: the Conquest of Color 19091954 (Alfred A. Knopf, New York, 2007) 